\title{
Evaluating the role of reference-genome phylogenetic distance on evolutionary inference
}

\author{
Aparna Prasad ${ }^{1}$, Eline D Lorenzen ${ }^{1}$, Michael V Westbury ${ }^{1 *}$
}

1. GLOBE Institute, University of Copenhagen, Øster Voldgade 5-7, Copenhagen, Denmark

Corresponding author: m.westbury@sund.ku.dk

\section{Abstract}

When a high-quality genome assembly of a target species is unavailable, an option to avoid the costly de novo assembly process is a mapping-based assembly. However, mapping shotgun data to a distant relative may lead to biased or erroneous evolutionary inference. Here, we used short-read data from a mammal and a bird species (beluga and rowi kiwi) to evaluate whether reference genome phylogenetic distance can impact downstream demographic (PSMC) and genetic diversity (heterozygosity, runs of homozygosity) analyses. We mapped to assemblies of species of varying phylogenetic distance (conspecific to genome-wide divergence of $>7 \%$ ), and de novo assemblies created using cross-species scaffolding. We show that while reference genome phylogenetic distance has an impact on demographic analyses, it is not pronounced until using a reference genome with $>3 \%$ divergence from the target species. When mapping to cross-species scaffolded assemblies, we are unable to replicate the original beluga demographic analyses, but can with the rowi kiwi, presumably reflecting the more fragmented nature of the beluga assemblies. As for genetic diversity estimates, we find that increased phylogenetic distance has a pronounced impact; heterozygosity estimates deviate incrementally as phylogenetic distance increases. Moreover, runs of homozygosity are removed when mapping to any non-conspecific assembly. However, these biases can be reduced when mapping to a cross-species scaffolded assembly. 
29 Taken together, our results show that caution should be exercised when selecting the reference genome for mapping assemblies. Cross-species scaffolding may offer a way to avoid a costly, traditional de novo assembly, while still producing robust, evolutionary inference.

\section{Introduction} assembly: mapping-based assemblies using a closely-related species as reference, or de novo assemblies. In the former approach, relatively little time and monetary expense is invested in sequencing one individual to high coverage ( $>20 x)$. After assembly, it is possible to make population-wide evolutionary inferences of the target species, including levels of genetic diversity and inbreeding, adaptive genomic changes, and demographic history (Barnett et al., 2020; Lord et al., 2020; Michael V. Westbury, Petersen, Garde, Heide-Jørgensen, \& Lorenzen, 2019).

Although mapping-based assemblies are less costly than de novo assemblies, there are some caveats. Biases towards the reference genome allele may be an issue when analysing population-level datasets. Such errors can arise during variant calling, when the alternative allele fails to be called altogether, or when heterozygous sites are incorrectly called as homozygous for the reference allele (Brandt et al., 2015; Ros-Freixedes et al., 2018). Although such issues are known to occur when mapping to a conspecific from a different population, biases caused by mapping to phylogenetically more distant taxa have only 
somewhat been addressed (Armstrong et al., 2020; M. V. Westbury et al., 2021). Problems

with correctly identifying variants may arise due to decreased mapping efficiency as reference-genome phylogenetic distance increases (Shapiro \& Hofreiter, 2014). However, the consequences of this on downstream analyses have yet to be comprehensively assessed. This leads to the question of whether the potentially costly de novo assembly process can be avoided when assemblies from phylogenetically more distant species are available. Insights into this will be especially important for the study of extinct species, where a conspecific reference genome is unlikely to be available (Barnett et al., 2020; Palkopoulou et al., 2018).

Here, we investigate the influence of the reference genome's phylogenetic distance to the target species on downstream evolutionary analyses. Specifically, we focused on estimates of (i) demographic history (reconstructed using PSMC), (ii) genetic diversity (genome-wide heterozygosity estimated using ANGSD, ROHan, SAMtools/BCFtools), (iii) inbreeding/runs of homozygosity (using ROHan). Additionally, we investigated whether biases can be overcome by using cross-species scaffolded con-specific assemblies as mapping reference.

We applied our methodology to two taxonomically disparate datasets; one based on mammals (beluga and incrementally divergent cetacean species), and one based on birds (rowi kiwi and incrementally divergent paleognath species). We selected these datasets based on the assumption that mammal and bird genomes may respond differently to reference biases, allowing for more generalised conclusions. Furthermore, while beluga whales are a relatively abundant species (Hobbs et al., 2019), rowi kiwi are threatened with extinction and have much lower population numbers (Robertson et al., 2017), which may play a role. 
Materials and methods

78

A simplified version of the methodologies implemented in this manuscript are

80

81

82

83

84

85

86

87

88

89

90

91

92

93

94

95

96

97

98

99

100

\section{Data}

For the cetacean comparative dataset, we downloaded the raw Illumina reads and an assembled genome of the beluga (Delphinapterus leucas, Genbank accession code:

GCF_002288925.2, SRA code: SRR5197961). In addition, we downloaded genome assemblies for five other cetacean species with varying genomic distance to the beluga (Table 1): narwhal (Monodon monoceros, Genbank accession code: GCF_005190385.1), narrowridged finless porpoise (Neophocaena asiaeorientalis, Genbank accession code:

GCF_003031525.1), bottlenose dolphin (Tursiops truncatus, Genbank accession code:

GCF_001922835.1), sperm whale (Physeter macrocephalus, Genbank accession code:

GCA_002837175.2), and minke whale (Balaenoptera acutorostrata, Genbank accession code: GCF_000493695.1). Assembly length, N50, and level of missing data for each assembly are listed in supplementary table $\mathrm{S} 1$.

For the paleognath comparative dataset, we downloaded raw reads and an assembled genome of the rowi kiwi (Genbank accession: GCF_003343035.1, SRA accession:

SRR6918118). We also downloaded published assemblies for three palaeognathae species of varying genomic distance to the rowi kiwi (Table 1): North Island brown kiwi (termed brown kiwi here, A. mantelli, Genbank accession: GCF_001039765.1), great spotted kiwi (termed spotted kiwi here, A. haastii, Genbank accession: GCA_003342985.1), and emu (Dromaius 
novaehollandiae, Genbank accession: GCF_003342905.1). Assembly length, N50, and levels of missing data for each assembly are listed in supplementary table S2.

103

\section{De novo and mapping assemblies}

All mappings were performed following the same procedure for the beluga/cetacean

106

107

108

For the beluga, we mapped the beluga short-read data to (i) published assemblies (beluga, narwhal, finless porpoise, bottlenose dolphin, sperm whale, minke whale), (ii) a de novo contig-level beluga assembly constructed for the purposes of this study, and (iii) five de novo beluga assemblies produced by cross-species scaffolding the contig-level assembly with each published non-beluga assembly using in-silico mate pair (MP) libraries.

(rowi kiwi, brown kiwi, spotted kiwi, emu), (ii) a de novo contig-level rowi kiwi assembly constructed for the purposes of this study, and (iii) three de novo rowi kiwi assemblies produced by cross-species scaffolding the contig-level assembly with each of the non-rowi kiwi published assembles using in-silico MP libraries. 

by first performing an error-correction step on the adapter-trimmed reads in tadpole from the BBtools toolsuite (Bushnell, 2014) and a kmer size of 31. We constructed the de novo assembly with the error-corrected reads using SOAPdenovo2 pregraph and contig (Luo et al., 2012), specifying a kmer size of 51, and otherwise using default parameters.

129 the seq-scripts pipeline. on the Genbank annotations.

\section{To construct the cross-species scaffolded assemblies we used the contig-level} assemblies from above and scaffolded them either five times independently in the case of the beluga, or three times independently in the case of the rowi kiwi. For this, we constructed insilico MP libraries using a modified version of the cross-species scaffolding pipeline (Grau, Hackl, Koepfli, \& Hofreiter, 2018) and repeat-masked versions of the published non-beluga assemblies (narwhal, finless porpoise, bottlenose dolphin, sperm whale, minke whale) and published non-rowi assemblies (brown kiwi, spotted kiwi, emu). Repeats were masked based

In short, we constructed a fasta consensus sequence using a consensus base-call approach (-doFasta 2) in ANGSD v0.931 (Korneliussen, Albrechtsen, \& Nielsen, 2014) and a minimum read depth of 3 (-minInddepth 3), minimum mapping quality of 25 (-minmapq 25), minimum base quality of 25 (-minq 25), and only considered reads that mapped uniquely to one location (-uniqueonly 1). We converted this fasta sequence into a pseudo-fastq sequence with a quality score of 40 for all covered bases using BBtools (Bushnell, 2014), as input for 
149 (https://github.com/thackl/seq-scripts), specifying a read length of 150bp and read depth of

We assessed the final assembly quality in the form of contiguity (N50) and amount of missing data using QUAST v4.5 (Gurevich, Saveliev, Vyahhi, \& Tesler, 2013).

\section{Sex-scaffold filtering}

To exclude sex-linked scaffolds in downstream demographic and heterozygosity analyses, we determined the scaffolds that likely originated from the sex-chromosomes for each of the scaffolded assemblies (published assemblies and the cross-species scaffolded assemblies) used in this study. We found putative sex-chromosome scaffolds in the cetacean 


\section{Divergence estimates}

To ensure comparability between the divergence estimates of our datasets, we calculated the autosome-wide divergence of our species of interest, either beluga or rowi kiwi, to the other species included in the study. To do this, we downloaded the raw reads for all the species (Supplementary table S3) and mapped them back to either the published beluga assembly or rowi kiwi assembly. We calculated the pairwise distance between species from the resultant mapped bam files and a consensus base call approach in ANGSD (-doIBS 2), and specifying the following parameters: -minq 25 -minmapq 25 -minind 4 -setMinDepthInd 5 -uniqueonly 1 -docounts 1 make a distance matrix (-makematrix 1), and only including autosomal scaffolds over 10kb in length (-rf).

\section{Demographic reconstruction}

To determine the influence of (a) phylogenetic distance of the reference genome to the target species, (b) reference genome contiguity, and (c) the utility of cross-species scaffolded reference genomes on demographic reconstruction, we ran a Pairwise Sequentially Markovian Coalescent model (PSMC) (Li \& Durbin, 2011) on each diploid genome, resulting in a total of twelve replicates for the beluga dataset and eight for the rowi kiwi dataset. We called diploid genome sequences using SAMtools and BCFtools v1.6 (Narasimhan et al., 2016), specifying a minimum quality score of 20 and minimum coverage of 10 .

We ran PSMC specifying atomic intervals $4+25 * 2+4+6$. Beluga PSMC outputs were plotted using a generation time of 32 years (Garde et al., 2015) and mutation rate of 1.65e-08 (Michael V. Westbury et al., 2019). To plot the rowi kiwi PSMC outputs, we calculated a mutation rate using the pairwise distance of the rowi kiwi to the brown kiwi (0.003123) and 
the formula pairwise distance $\mathrm{x} 2$ / divergence time. We used a divergence time $\sim 3.8 \mathrm{Ma}$ (De

\section{Genetic diversity}

To determine the influence of (a) phylogenetic distance of the reference genome to the

heterozygosity of the beluga mapped to all twelve cetacean assemblies and the rowi kiwi mapped to all eight paleognath assemblies.

We calculated heterozygosity for each of the datasets using ANGSD. We estimated account with the GATK algorithm (-GL 2), and specifying the following filters: only include sites with a read depth of at least 5 (-mininddepth 5), minimum mapping and base qualities of 30 (-minmapq 30, -minq 30), only include reads mapping uniquely to one location (include autosomal scaffolds (-rf), and the extended adjust quality scores around indels

214 parameter (-baq 2). Heterozygosity was computed from the output of this using realSFS from 215 the ANGSD toolsuite, specifying 20 megabase pairs (Mb) windows of covered sites (-nSites). 
assessed the influence of parameter selection in ANGSD on heterozygosity estimates by computing heterozygosity using the procedure outlined above, but replacing the 'extended adjust quality scores around indels' parameter (-baq 2), with (i) adjust quality scores around insertion/deletions (indels) (-baq 1), (ii) no indel quality score adjustment (-baq 0), or (iii) extended adjust quality scores around indels (-baq 2), and adjust quality for reads with multiple mismatches to the reference (-C 50).

To assess whether software choice can impact results, we used two additional methods to compute heterozygosity of the beluga mapped to the six published assemblies: ROHan file (SAMtools/BCFtools).

In ROHan, we used default parameters to calculate autosome-wide levels of window as being a $\mathrm{ROH}$, if the window has an average heterozygosity of less than 1e-5. To calculate average autosome-wide heterozygosity from the diploid file used for the PSMC analysis, we used seqtk comp (https://github.com/lh3/seqtk).

\section{Inbreeding (runs of homozygosity)}

As ROHan simultaneously outputs runs of homozygosity as well as autosome-wide

239 levels of heterozygosity, we could evaluate how reference genome phylogenetic distance

240 influences perceived inbreeding estimates using $\mathrm{ROH}$. We did not retrieve any significant

$241 \mathrm{ROH}$ in the beluga dataset using ROHan, so we were unable to investigate this further using 
the beluga data. We repeated the above ROHan analysis using the rowi kiwi mapped to all eight assemblies (both published and cross-species scaffolded).

\section{Results}

Mapping

Mapping results of the beluga raw reads to each cetacean assembly can be found in distance to the reference genome increases, there is a general trend of a decreasing number of unique reads mapping. This trend is not seen when mapping to the five and three crossspecies scaffolded assemblies for beluga and rowi kiwi, respectively. However, less reads map to these assemblies than to the conspecific assemblies.

\section{Cross-species scaffolded de novo assemblies} assemblies were more contiguous, with N50s ranging from $283 \mathrm{~kb}$ to $614 \mathrm{~kb}$ (Supplementary

261 missing data rates of $0.5 \%$ - 6\% (Supplementary table S1). 
data $(10 \%-21 \%)$. However, this was comparable to the brown kiwi assembly with $14 \%$ data.

Assembly contiguities were also more comparable to the published assemblies, which had

$\mathrm{N} 50 \mathrm{~s}$ of $1.4 \mathrm{Mb}-5.7 \mathrm{Mb}$ (Supplementary table S2).

\section{Demographic reconstruction}

Beluga - With increasing phylogenetic distance of the reference genome, we see an

incremental increase in deviation from the pattern obtained when mapping to the published

beluga assembly (Fig 2A). However, we do not see an incremental change when using our

five cross-species scaffolded assemblies as reference. Instead we see that all newly assembled genomes produce the same PSMC output. However, this output differs from the pattern obtained when mapping to the published beluga assembly (Fig 2B).

When comparing PSMC results produced by mapping to the published beluga

279

280

assembly, and by mapping to our de novo contig-level beluga assembly, we see a pattern of increase in $\mathrm{N}_{\mathrm{e}} \sim 500$ thousand years ago (kya) followed by a decrease $\sim 150$ kya. This is consistent between both assemblies. However, the values of effective population size $\left(\mathrm{N}_{\mathrm{e}}\right)$ are much lower when mapping to the de novo contig-level assembly (Supplementary fig S1).

$$
\text { Rowi kiwi - Unlike the beluga, the PSMC results of the rowi kiwi were vastly }
$$
different when mapping to phylogenetic distant references compared to the published rowi assembly (Supplementary fig S2). However, we do see the incremental change as phylogenetic distance increases when mapping to the non-rowi assemblies. We investigated if there was a problem with the published rowi assembly by reassembling it using the published short-read and $3 \mathrm{~kb}$ mate-paired libraries (Sackton et al., 2019) with SOAPdenovo. Our 
reassembled rowi kiwi genome was much less contiguous than the published version $(0.3 \mathrm{Mb}$

vs $1.7 \mathrm{Mb}$ ) and had more missing data (12.7\% vs. 1.6\%). However, the PSMC produced when

mapping to this assembly was much more consistent with what we would have expected

based on the beluga results, and shows a demographic history similar to when mapping to the

assemblies from the other three non-rowi kiwi species (Fig 3A). Hence, we only considered

this re-assembly when assessing the inference of reference genome on demographic history

results in the rowi kiwi. The results produced after mapping to the cross-species scaffolded

rowi kiwi assemblies are much more similar to those from the re-assembled published rowi

kiwi assembly (Fig 3B).

genome, and by mapping to the contig-level assembly, we see similar general trajectories.

However, the values of effective population size $(\mathrm{Ne})$ are much lower when mapping to the de novo contig-level assembly as seen in the beluga (Supplementary fig S3).

\section{Genetic diversity}

When using ANGSD, ROHan, and SAMtools/BCFtools, we see a general trend of

307 increasing heterozygosity as reference genome phylogenetic distance increases. Which is also consistent when applying the alternative ANGSD parameter sets (i) -baq 1 instead of -baq 2, and (ii) -baq 0 instead of -baq 2 (Fig 4A,B, Supplementary figs S4 and S5, Supplementary tables S10 and S11). In contrast, when using ANGSD parameter set (iii) adjusted for reads with multiple mismatches to the reference (-C 50), we see a general trend of decreasing 
When using the cross-species scaffolded assemblies as reference genomes, we obtain results more comparable to those obtained when using the published conspecific assemblies as reference (Fig 4C,D). However, we do not see this when using SAMtools/BCFtools and the beluga dataset. Instead, we observe a decrease in heterozygosity relative to the published conspecific beluga assembly. The decrease is of a similar magnitude regardless of which cetacean species was used for scaffolding (Supplementary table S12). compared to a scaffolded assembly (Supplementary fig S7). This same pattern was also seen when comparing the de novo contig-level rowi kiwi assembly to our reassembled version, but not compared to the published assembly (Supplementary fig S8).

\section{Inbreeding}

When mapping the beluga reads to any reference genome (including the published conspecific beluga assembly), we did not uncover any ROH. When running ROHan on the rowi kiwi mapped to a published conspecific rowi kiwi assembly, we uncovered $\mathrm{ROH}$, but not when mapping to any of the non-rowi kiwi assemblies (Table 2).

\section{Discussion}

Through a detailed comparison of results produced after mapping to multiple reference 
inferences. In general, as the phylogenetic distance of the reference genome increases, results become incrementally less reliable with regards to demographic history, genetic diversity, and inbreeding estimates.

With regards to demographic history analyses using PSMC, phylogenetic distance of

343 the reference genome to the target species did not appear to affect the overall trajectories, but

344 did result in relatively decreased $\mathrm{N}_{\mathrm{e}}$ estimates. However, this only became apparent when

345 using a reference genome more than $0.14 \%$ different to the target species (e.g. beluga vs

346 finless porpoise) (Figs 2A, 3A). Based on these results, if a conspecific assembly is not

347 available, using an assembly from a relatively closely-related species is unlikely to interfere

348 with the overall demographic trajectory.

In contrast with the demographic results, the bias that reference-genome selection

352 heterozygous sites to be incorrectly called as homozygous for the reference allele (Brandt et 353 al., 2015; Ros-Freixedes et al., 2018). However, we see a general increase in heterozygosity, as opposed to the expected decrease (Fig 4). Therefore, misalignments may be a larger factor in falsely calling heterozygous alleles as opposed to simply incorrect base calling. When applying a strict filter that corrects for reads with multiple mismatches to the reference

357 genome (-C 50), it may be possible to eliminate increased heterozygosity due to

358 misalignments (Supplementary figure S6). However, this is still associated with issues, as we observed a general decrease in heterozygosity due to putatively incorrect basecalls. 
ROH (Table 2). When mapping to a non-conspecific reference genome, we observed a

the reference increases, this could artificially increase the heterozygosity level in ROH, making the ROH no longer observable. novo assembly is that it only requires a single lane of Illumina sequencing, and an available assembly from a closely-related species. However, at least in the case of the beluga dataset, it can result in much more fragmented assemblies (Supplementary tables S8), and may therefore not always be applicable, especially when highly contiguous assemblies are required (e.g. for PSMC and ROH analyses). Nevertheless, using cross-species scaffolded assemblies as reference resulted in relatively reliable PSMC and ROH results for the rowi kiwi (Fig 3, Table may be more reliable when heterozygous sites do not have the perfect near-50/50 allele ratios. 
assemblies (Supplementary table S8). The inability to produce contiguous scaffolds like the

390 (Weisenfeld et al., 2014) to ensure the insert sizes are uniform may improve the contiguity of the final assembly, and make results reliant on highly contiguous data, such as PSMC, more reliable.

Although the assemblies are more fragmented, this does not mean they are completely devoid of information for the PSMC analysis. Results using the cross-species scaffolded assemblies still present the increase in $\mathrm{N}_{\mathrm{e}} \sim 500$ kya and decrease $\sim 150$ kya seen when using

Furthermore, when comparing PSMC results produced via mapping to the scaffold-level and contig-level assemblies, we also see a similar pattern of population size change, but with

400 different values of $\mathrm{N}_{\mathrm{e}}$ (Supplementary figs S1 and S3). This suggests that contiguity may not 401 influence the pattern as much as the scale, and may still be useful for investigating relative changes in $\mathrm{N}_{\mathrm{e}}$ rather than absolute values of $\mathrm{N}_{\mathrm{e}}$ itself.

Our analyses uncovered a potential problem with the published rowi kiwi assembly.

405 When comparing results mapped to the published assembly against non-conspecific assemblies, cross-species scaffolded assemblies, and a reassembly of the published data, we

407 uncover large discrepancies in the results, especially in the PSMC results (Supplementary figs 408 S2 and S3). As our assemblies all used the same published raw data, we suspect that these 
409

410

411

413

414

415

416

417

418

419

420

421

422

423

424

425

426

427

428

429

430

431

discrepancies resulted from miss-assemblies during the original de novo assembly process in Allpaths-LG (Butler et al., 2008). Although outside of the scope of the present study, these results show that caution should be exercised in reference genome selection for mapping assemblies; if multiple assemblies are available, it may be beneficial to test robustness of results against multiple reference genomes.

Taken together, our results show that demographic analyses of a single individual mapped to a phylogenetically distant reference genome may be considered reliable with regards to demographic trajectories (as in relative changes in $\mathrm{N}_{\mathrm{e}}$, rather than absolute values of $\mathrm{N}_{\mathrm{e}}$ ). However, the phylogenetic distance of the reference genome can lead to overestimation of heterozygosity and, in turn, underestimations of ROH. Finally, if no assembly from a suitably closely related species is available as a mapping reference, crossspecies scaffolded assemblies appear to be a valid and likely more suitable option for evolutionary inference.

\section{Acknowledgements}

The work was also supported by the Independent Research Fund Denmark | Natural Sciences, Forskningsprojekt 1, grant no. 8021-00218B to EDL.

\section{References}

Armstrong, E. E., Taylor, R. W., Miller, D. E., Kaelin, C. B., Barsh, G. S., Hadly, E. A., \& Petrov, D. (2020). Long live the king: chromosome-level assembly of the lion (Panthera leo) using linked-read, Hi-C, and long-read data. BMC Biology, 18(1), 3. 
Barnett, R., Westbury, M. V., Sandoval-Velasco, M., Vieira, F. G., Jeon, S., Zazula, G., ... Gilbert, M. T. P. (2020). Genomic Adaptations and Evolutionary History of the Extinct Scimitar-Toothed Cat, Homotherium latidens. Current Biology: CB, 30, 1-8.

Brandt, D. Y. C., Aguiar, V. R. C., Bitarello, B. D., Nunes, K., Goudet, J., \& Meyer, D. (2015). Mapping Bias Overestimates Reference Allele Frequencies at the HLA Genes in the 1000 Genomes Project Phase I Data. G3 , 5(5), 931-941.

Bushnell, B. (2014). BBTools software package. URL Http://sourceforge. Net/projects/bbmap.

Butler, J., MacCallum, I., Kleber, M., Shlyakhter, I. A., Belmonte, M. K., Lander, E. S., ... Jaffe, D. B. (2008). ALLPATHS: de novo assembly of whole-genome shotgun microreads. Genome Research, 18(5), 810-820.

De Cahsan, B., \& Westbury, M. V. (2020). Complete mitochondrial genomes offer insights into the evolutionary relationships and comparative genetic diversity of New Zealand's iconic kiwi (Apteryx spp.). New Zealand Journal of Zoology, 1-9.

Garde, E., Hansen, S. H., Ditlevsen, S., Tvermosegaard, K. B., Hansen, J., Harding, K. C., \& Heide-Jørgensen, M. P. (2015). Life history parameters of narwhals (Monodon monoceros) from Greenland. Journal of Mammalogy, 96(4), 866-879.

Grabherr, M. G., Russell, P., Meyer, M., Mauceli, E., Alföldi, J., Di Palma, F., \& LindbladToh, K. (2010). Genome-wide synteny through highly sensitive sequence alignment: Satsuma. Bioinformatics , 26(9), 1145-1151.

Grau, J. H., Hackl, T., Koepfli, K.-P., \& Hofreiter, M. (2018). Improving draft genome contiguity with reference-derived in silico mate-pair libraries. GigaScience, 7(5), giy029. Gurevich, A., Saveliev, V., Vyahhi, N., \& Tesler, G. (2013). QUAST: quality assessment tool for genome assemblies. Bioinformatics , 29(8), 1072-1075. 
Hobbs, R. C., Reeves, R. R., Prewitt, J. S., Desportes, G., Breton-Honeyman, K., Christensen, T., ... Watt, C. A. (2019). Global Review of the Conservation Status of Monodontid Stocks. Marine Fisheries Review, 81, 1+.

Jiang, H., Lei, R., Ding, S.-W., \& Zhu, S. (2014). Skewer: a fast and accurate adapter trimmer for next-generation sequencing paired-end reads. BMC Bioinformatics, 15, 182.

Korneliussen, T. S., Albrechtsen, A., \& Nielsen, R. (2014). ANGSD: Analysis of Next Generation Sequencing Data. BMC Bioinformatics, 15, 356.

Li, H., \& Durbin, R. (2009). Fast and accurate short read alignment with Burrows-Wheeler transform. Bioinformatics , 25(14), 1754-1760.

Li, H., \& Durbin, R. (2011). Inference of human population history from individual wholegenome sequences. Nature, 475(7357), 493-496.

Li, H., Handsaker, B., Wysoker, A., Fennell, T., Ruan, J., Homer, N., .. 1000 Genome Project Data Processing Subgroup. (2009). The Sequence Alignment/Map format and SAMtools. Bioinformatics , 25(16), 2078-2079.

Lord, E., Dussex, N., Kierczak, M., Díez-Del-Molino, D., Ryder, O. A., Stanton, D. W. G., ... Dalén, L. (2020). Pre-extinction Demographic Stability and Genomic Signatures of Adaptation in the Woolly Rhinoceros. Current Biology: CB, 30(19), 3871-3879.e7.

Luo, R., Liu, B., Xie, Y., Li, Z., Huang, W., Yuan, J., ... Wang, J. (2012). SOAPdenovo2: an empirically improved memory-efficient short-read de novo assembler. GigaScience, $1(1), 18$.

Narasimhan, V., Danecek, P., Scally, A., Xue, Y., Tyler-Smith, C., \& Durbin, R. (2016). BCFtools/RoH: a hidden Markov model approach for detecting autozygosity from nextgeneration sequencing data. Bioinformatics, 32(11), 1749-1751.

Palkopoulou, E., Lipson, M., Mallick, S., Nielsen, S., Rohland, N., Baleka, S., ... Reich, D. 
(2018). A comprehensive genomic history of extinct and living elephants. Proceedings of the National Academy of Sciences of the United States of America, 115(11), E2566E2574.

Renaud, G., Hanghøj, K., Korneliussen, T. S., Willerslev, E., \& Orlando, L. (2019). Joint Estimates of Heterozygosity and Runs of Homozygosity for Modern and Ancient Samples. Genetics, 212(3), 587-614.

Robertson, H. A., Baird, K., Dowding, J. E., Elliott, G. P., Hitchmough, R. A., Miskelly, C. M., ... Taylor, G. A. (2017). Conservation status of New Zealand birds, 2016 (p. 23). Department of Conservation, Wellington: New Zealand Threat Classification Series 19. Ros-Freixedes, R., Battagin, M., Johnsson, M., Gorjanc, G., Mileham, A. J., Rounsley, S. D., \& Hickey, J. M. (2018). Impact of index hopping and bias towards the reference allele on accuracy of genotype calls from low-coverage sequencing. Genetics, Selection, Evolution: GSE, 50(1), 64.

Sackton, T. B., Grayson, P., Cloutier, A., Hu, Z., Liu, J. S., Wheeler, N. E., ... Edwards, S. V. (2019). Convergent regulatory evolution and loss of flight in paleognathous birds. Science, 364(6435), 74-78.

Shapiro, B., \& Hofreiter, M. (2014). A paleogenomic perspective on evolution and gene function: new insights from ancient DNA. Science, 343(6169), 1236573.

Weir, J. T., Haddrath, O., Robertson, H. A., Colbourne, R. M., \& Baker, A. J. (2016). Explosive ice age diversification of kiwi. Proceedings of the National Academy of Sciences of the United States of America, 113(38), E5580-E5587.

Weisenfeld, N. I., Yin, S., Sharpe, T., Lau, B., Hegarty, R., Holmes, L., ... Jaffe, D. B. (2014). Comprehensive variation discovery in single human genomes. Nature Genetics, 46(12), 1350-1355. 
504 Westbury, M. V., Le Duc, D., Duchêne, D. A., Krishnan, A., Prost, S., Rutschmann, S., ... Hyaenidae. Molecular Biology and Evolution, msab055.

Westbury, M. V., Petersen, B., Garde, E., Heide-Jørgensen, M. P., \& Lorenzen, E. D. (2019). Abundance Size. iScience, 15, 592-599.

\section{Author contributions}

Conceptualization, MVW; Formal analysis, AP, MVW; Writing - Original Draft MVW; 
Tables

Table 1: Genome-wide pairwise divergence estimates of the species used in this study.

\begin{tabular}{|l|r|r|}
\hline Species & Compared to & Divergence \\
\hline Narwhal & Beluga & 0.0050 \\
\hline Finless porpoise & Beluga & 0.0143 \\
\hline Bottlenose dolphin & Beluga & 0.0202 \\
\hline Sperm whale & Beluga & 0.0318 \\
\hline Minke whale & Beluga & 0.0344 \\
\hline Brown kiwi & Rowi kiwi & 0.0031 \\
\hline Spotted kiwi & Rowi kiwi & 0.0079 \\
\hline Emu & Rowi kiwi & 0.0734 \\
\hline
\end{tabular}

Table 2: Autosomal heterozygosity and runs of homozygosity (ROH) estimates of the rowi kiwi when mapped to a variety of different reference genomes. Reference genomes named 'Rowi -' are constructed using cross-species scaffolding and the species depicted after the hyphen.

\begin{tabular}{|l|l|l|l|l|l|}
\hline Reference genome & $\begin{array}{l}\text { Global } \\
\text { heterozygosity } \\
\text { rate }\end{array}$ & Lower limit & Upper limit & $\begin{array}{l}\text { Segments in } \\
\text { ROH (\%) }\end{array}$ & $\begin{array}{l}\text { Avg. } \\
\text { length of } \\
\text { ROH (bp) }\end{array}$ \\
\hline Rowi (published) & 0.00121 & 0.00111 & 0.00126 & 4.42 & $1,644,440$ \\
\hline Rowi (re-assembled) & 0.00105 & 0.00086 & 0.00123 & 1.57 & $1,076,920$ \\
\hline Brown kiwi & 0.00121 & 0.00107 & 0.00139 & 0.22 & $1,000,000$ \\
\hline Spotted kiwi & 0.00119 & 0.00108 & 0.00134 & 0.00 & 0 \\
\hline Emu & 0.00177 & 0.00157 & 0.00193 & 0.00 & 0 \\
\hline Rowi - brown kiwi & 0.00107 & 0.00097 & 0.00116 & 2.74 & $1,833,330$ \\
\hline Rowi - spotted kiwi & 0.00106 & 0.00098 & 0.00117 & 2.82 & $1,444,440$ \\
\hline Rowi - emu & 0.00099 & 0.00089 & 0.00107 & 3.28 & $1,558,140$ \\
\hline
\end{tabular}



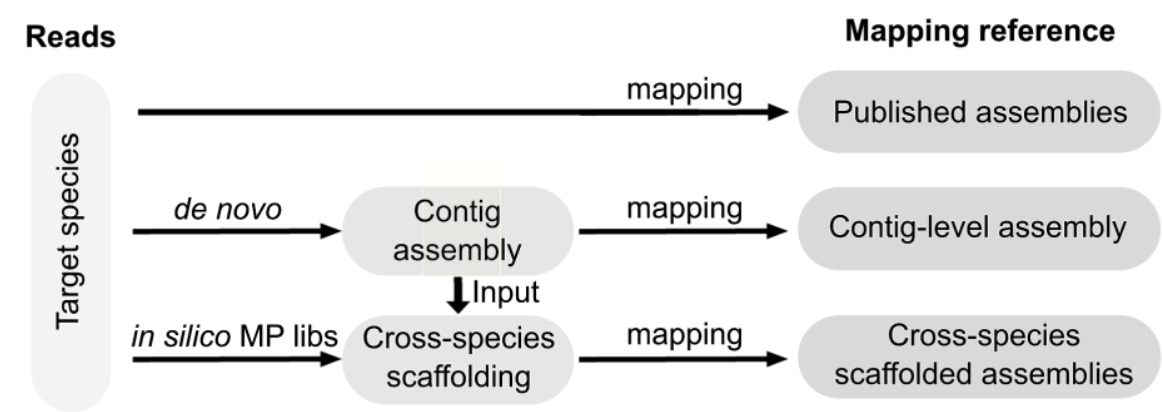

Analyses

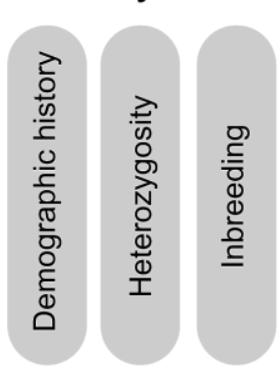

557 Figure 1: Overview of the approaches used to investigate the role reference genome plays in 558 downstream demographic history and genetic diversity results. Raw reads are mapped to

559 published assemblies, a de novo contig-level assembly, or cross-species scaffolded

560 assemblies. Contig-level assemblies are constructed using the raw reads. Cross-species

561 scaffolded assemblies are made by scaffolding the contig assembly using in-silico mate-pair 562 (MP) libraries. 


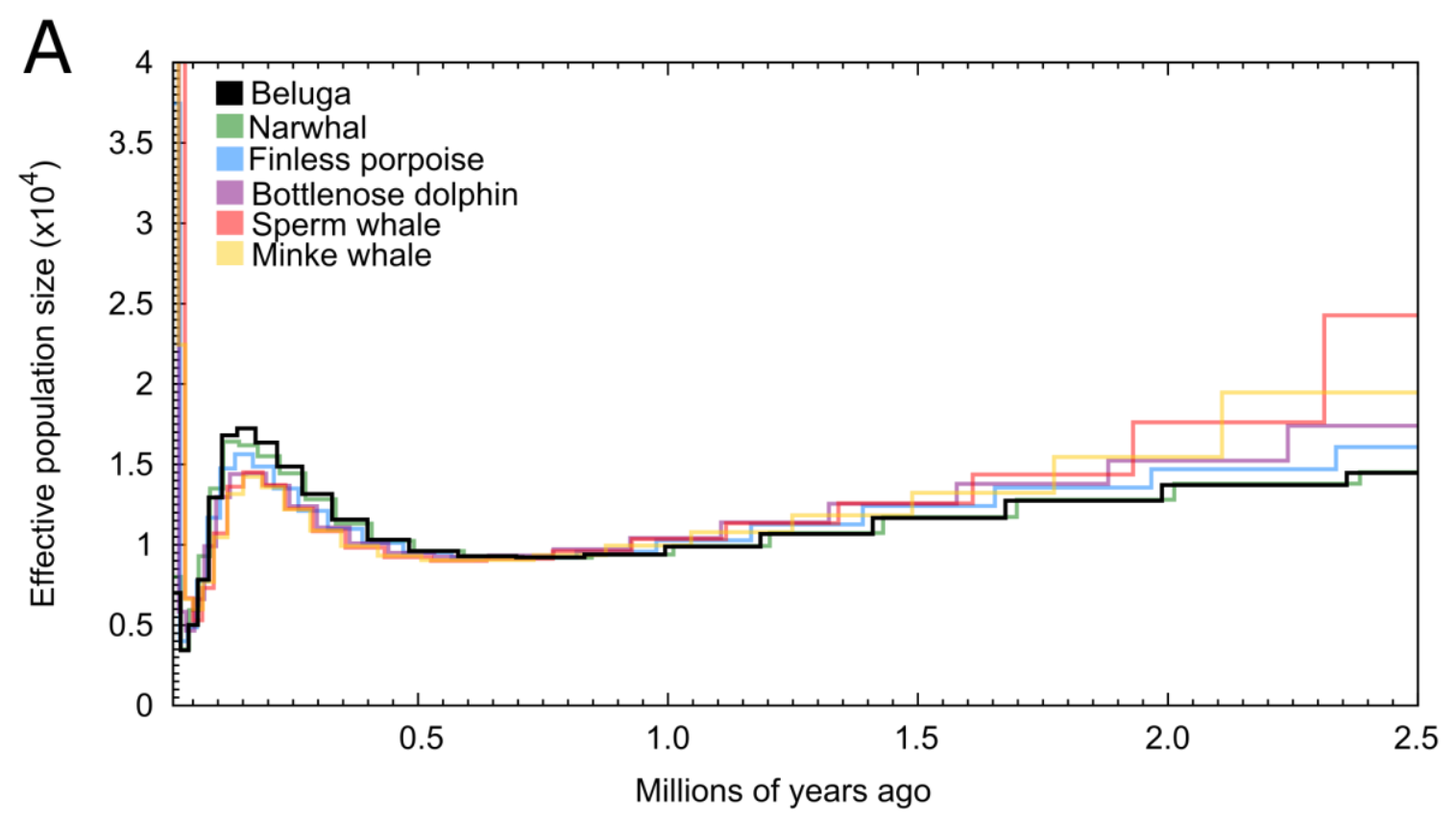

B

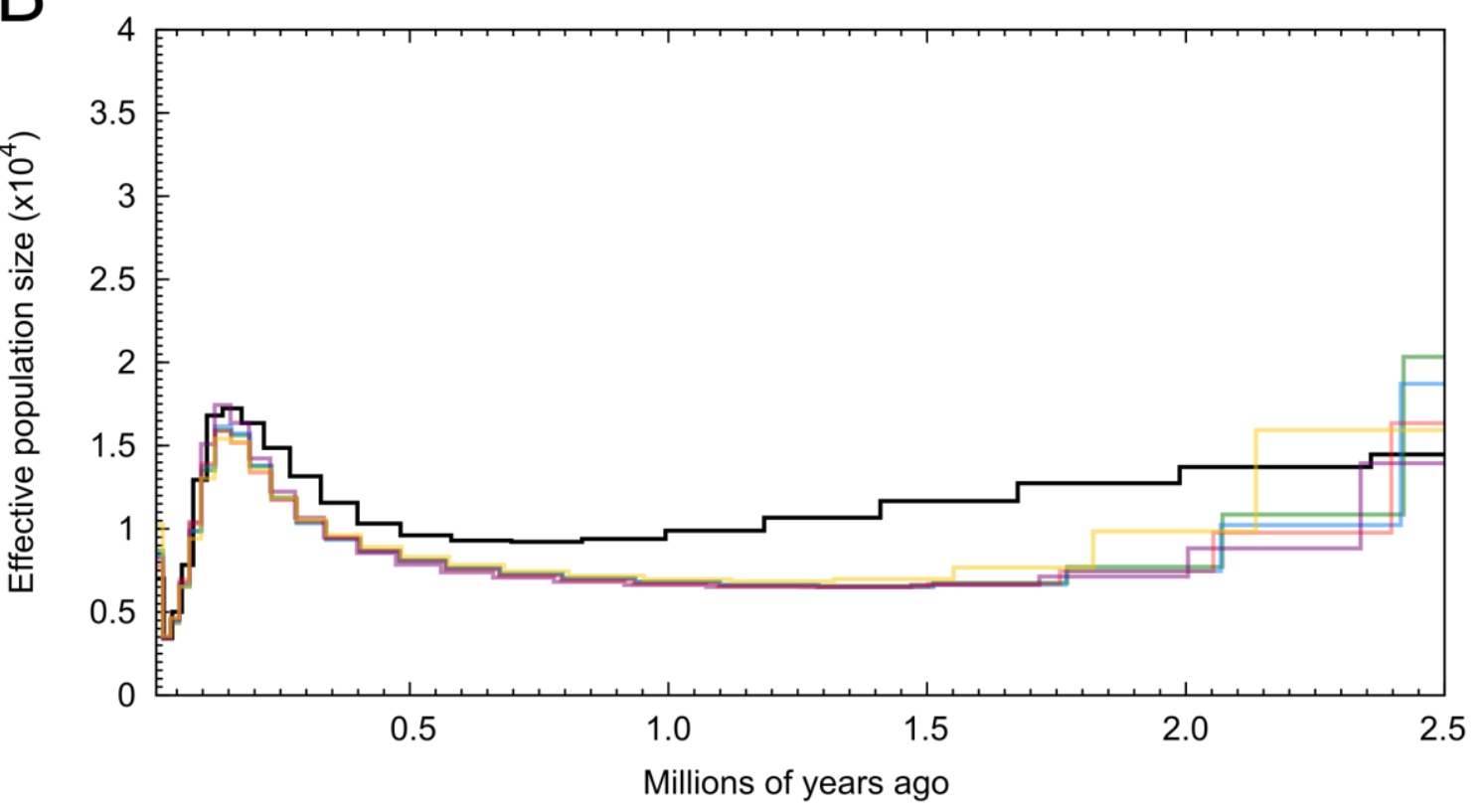

Figure 2: Beluga demographic history over the last 2.5 million years. Demographic trajectories in each panel represent genomes generated by mapping beluga reads to (A)

568 assemblies of six different phylogenetically distant species (including a conspecific), colours 569 indicate species of the reference genome, and (B) de novo assemblies constructed using cross570 species scaffolding and the published beluga assembly, colours show the species used to 571 scaffold the de novo beluga contig-level assembly. 

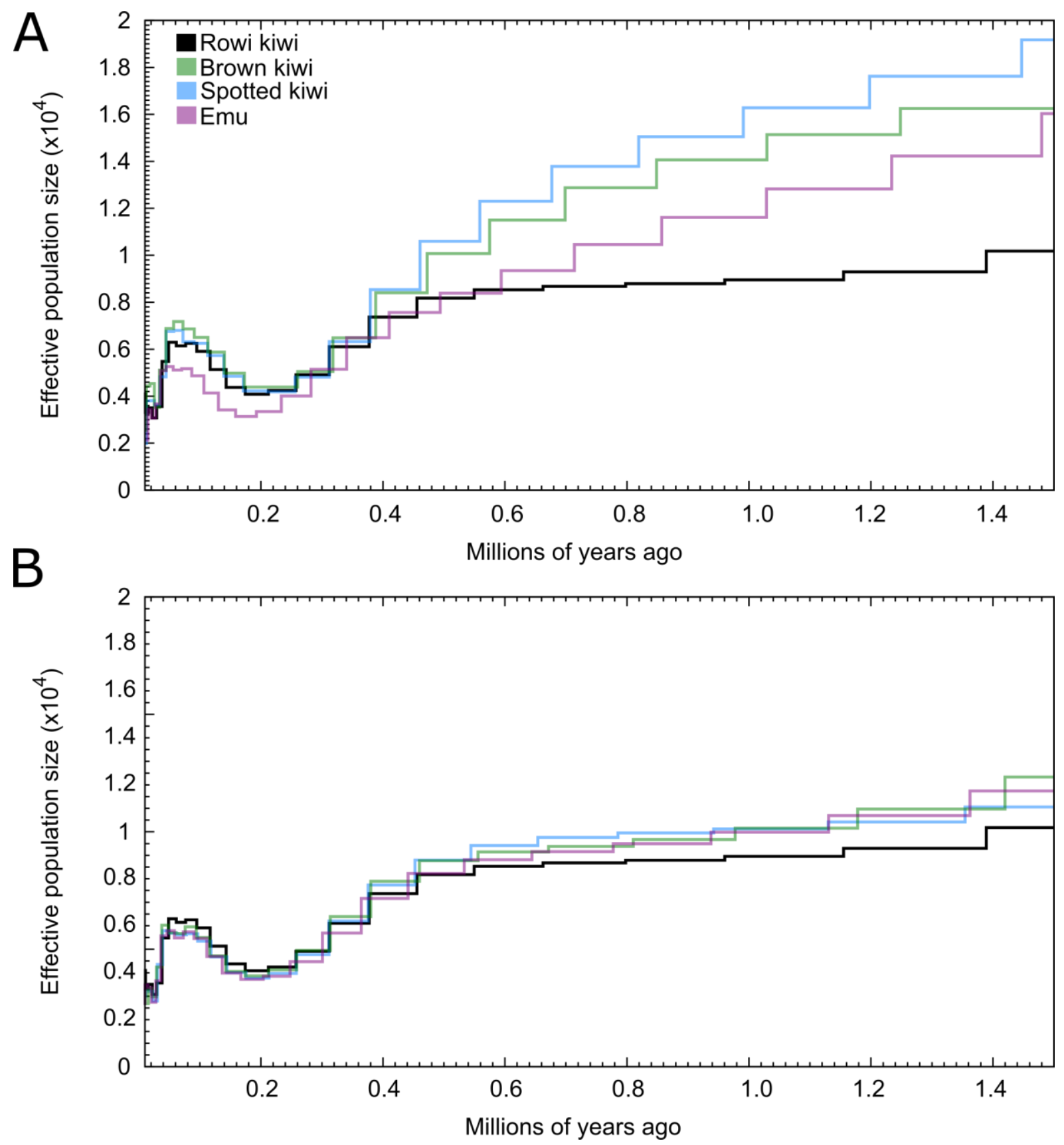

Figure 3: Rowi kiwi demographic history over the last 1.5 million years. Demographic

574 trajectories in each panel represent genomes generated by mapping rowi kiwi reads to (A)

575 assemblies of four different phylogenetically distant species (including our re-assembled rowi

576 kiwi assembly), colours indicate species of the reference genome, and (B) de novo rowi kiwi

577 assemblies constructed using cross-species scaffolding and our reassembled rowi kiwi

578 assembly - colours show the species used to scaffold the de novo rowi kiwi contig-level

579 assembly. 

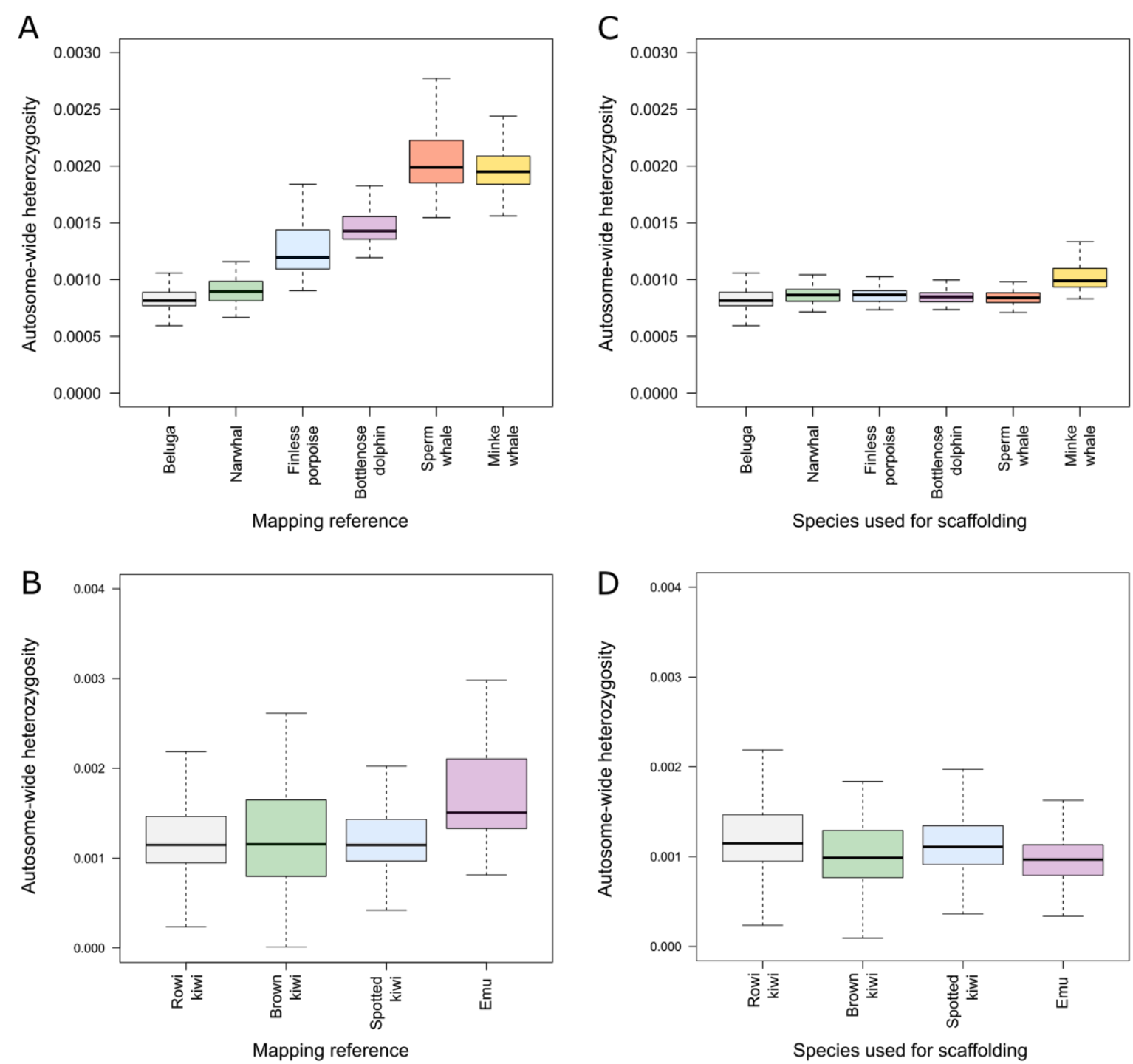

Figure 4: Autosome-wide heterozygosity estimates of the beluga and rowi kiwi mapped to different reference genomes. A single beluga individual was mapped to (A) six downloaded assemblies, and (B) a published beluga assembly and de novo beluga assemblies constructed using cross-species scaffolding. A single rowi kiwi individual was mapped to (C) our reassembled rowi kiwi genome and the three downloaded non-rowi kiwi assemblies, and (D) de novo rowi kiwi assemblies constructed using either the published rowi kiwi mate-pair libraries or using cross-species scaffolding with mate-pair (MP) libraries constructed from each of the three non-rowi kiwi assemblies. 